\title{
Hypertension and Pregnancy: Mechanisms and Management
}

\author{
${ }^{1}$ Mie Saiki, ${ }^{2}$ Vesna D Garovic
}

\begin{abstract}
Hypertensive pregnancy disorders encompass a spectrum of conditions, including preeclampsia, a multisystem hypertensive disease that is unique to pregnancy, eclampsia, and gestational and chronic hypertension. Severe forms of preeclampsia, including its convulsive form - eclampsia - represent obstetrical emergencies, the therapy of which is immediate delivery. The goal of antihypertensive therapy in these patients is to prevent maternal cardiac, cerebrovascular, and renal complications. Central to the medical management of hypertension in pregnancy is the careful balance between maternal benefits from improved blood pressure control and fetal risks from intrauterine drug exposure and changes in uteroplacental perfusion. Women with chronic hypertension and hypertension onset before pregnancy should undergo prepregnancy counseling regarding medication safety during pregnancy and evaluation for end-organ damage, which will help define their blood pressure goals during pregnancy. Women with gestational hypertension (hypertension onset in the second half of pregnancy) require close monitoring for signs of progression to preeclampsia. Adequate care of these patients relies on regular follow-ups, judicious use of antihypertensive medications, and close monitoring for early signs of preeclampsia. While these patients are typically cared for by high-risk obstetricians, input from internists and related subspecialties increasingly is being recognized as important for optimization of patient blood pressure treatment and overall pregnancy outcomes.
\end{abstract}

Keywords: Blood pressure management, Hypertensive pregnancy disorders, Preeclampsia.

How to cite this article: Saiki M, Garovic VD. Hypertension and Pregnancy: Mechanisms and Management. Hypertens J 2016;2(3):131-138.

\section{Source of support: Nil \\ Conflict of interest: None}

\section{HYPERTENSIVE DISORDERS OF PREGNANCY: CLASSIFICATION, PRESENTATION, AND MECHANISMS}

Hypertensive pregnancy disorders affect $10 \%$ of pregnancies and include preeclampsia, eclampsia, and

\footnotetext{
${ }^{1}$ Research Trainee, ${ }^{2}$ Professor

${ }^{1,2}$ Division of Nephrology and Hypertension, Department of Medicine, Mayo Clinic College of Medicine, Rochester Minnesota, USA

Corresponding Author: Vesna D Garovic, Division of Nephrology and Hypertension, Department of Medicine, Mayo Clinic College of Medicine, Rochester, Minnesota, USA, Phone: 5072661963, e-mail: garovic.vesna@mayo.edu
}

chronic and gestational hypertension (Table 1). In 2000, the Working Group of the National High Blood Pressure Education Program (NHBPEP) defined hypertension in pregnancy as a blood pressure $\geq 140 \mathrm{~mm} \mathrm{Hg}$ systolic and/ or $\geq 90 \mathrm{~mm} \mathrm{Hg}$ diastolic on two occasions, at least 6 hours apart. ${ }^{1}$ For the diagnosis of preeclampsia, both hypertension and proteinuria ( $300 \mathrm{mg}$ or greater in a 24 -hour urine specimen, or 1+ dipstick) were required. The American College of Obstetricians and Gynecologists (the College) assembled a task force of specialists in the management of hypertension in pregnancy in 2013 to issue evidence-based recommendations for clinical practice. ${ }^{2}$ With respect to the diagnostic criteria for preeclampsia, the most notable changes made by the College related to the importance of proteinuria, which now is neither mandatory for the diagnosis of preeclampsia, nor is it considered a marker of preeclampsia severity. Based on these recommendations, the diagnosis of preeclampsia can be entertained in the absence of proteinuria on the basis of clinical and laboratory abnormalities in women

Table 1: Hypertension in pregnancy: Classification and definitions

Preeclampsia Preeclampsia is a pregnancy-specific disorder characterized by hypertension (blood pressure $\geq 140 / 90 \mathrm{~mm} \mathrm{Hg}$ ) and proteinuria of $300 \mathrm{mg}$ or greater in a 24-hour urine. In the absence of proteinuria, preeclampsia can be diagnosed as hypertension in association with a variety of features including thrombocytopenia, impaired liver function tests, the new development of renal insufficiency, pulmonary edema, or newonset cerebral or visual disturbances. Eclampsia is the convulsive form of preeclampsia that affects $0.1 \%$ of all pregnancies.

Gestational Hypertension occurring for the first time during hypertension the second half of pregnancy in the absence of proteinuria. It includes (i) women with preeclampsia who have not yet developed proteinuria, (ii) transient hypertension: Those with hypertension alone, with blood pressure returning to normal by 12 weeks postpartum, and (iii) chronic hypertension: A subset of patients in whom blood pressure remains elevated after 12 weeks postpartum.

Chronic Blood pressure $\geq 140 / 90 \mathrm{~mm} \mathrm{Hg}$ prior to hypertension pregnancy or before the 20th week of gestation. Preeclampsia Up to $30 \%$ of women with chronic hypertension superimposed develop preeclampsia, commonly heralded on chronic by proteinuria, which occurs for the first time hypertension during the third trimester. 
with new-onset hypertension (Table 1). Common laboratory tests for the diagnosis of and differential diagnosis among the hypertensive pregnancy disorders include urinalysis, hemoglobin, hematocrit, platelet count, serum uric acid, albumin, and kidney and liver function tests.

Preeclampsia, unlike the other hypertensive disorders of pregnancy, is a systemic disease, commonly associated with proteinuria. It occurs after 20 weeks of gestation and affects approximately $5 \%$ of all pregnancies. Together with its related conditions, namely eclampsia, its convulsive form, and HELLP syndrome (an acronym for Hemolysis, Elevated Liver enzymes, and Low Platelet count), ${ }^{3}$ preeclampsia remains one of the leading causes of fetal and maternal morbidity and mortality. Preeclampsia traditionally has been considered a disease of first pregnancy. However, women with a history of preeclampsia are at increased risk during their subsequent pregnancies. Several other risk factors are well recognized, which may aid in the early recognition of patients at risk (Table 2). Preeclampsia may develop de novo in previously healthy pregnant women or occur in women with preexisting conditions, such as renal disease or chronic hypertension, i.e., superimposed preeclampsia. Maternal complications occur as a consequence of hypertensive end-organ damage of the central nervous system (stroke), kidneys (acute renal failure), and heart (acute cardiac decompensation). In a recent study examining all maternal deaths after the 20th week of pregnancy, preeclampsia and eclampsia were responsible for 790 of 4,024 deaths between 1979 and 1992. ${ }^{4}$ The overall preeclampsia-eclampsia case-fatality rate was 6.4 cases per 10,000 cases at delivery. The burden is even higher in developing countries. The etiology of this condition remains elusive; thus, specific screening, preventive, and treatment strategies are not available.

\section{Pathophysiology of Preeclampsia}

The role of maternal vascular adaptation and placental angiogenesis in normal pregnancy has been well recognized and studied. The fact that hypertension rapidly resolves upon the removal of the products of conception

Table 2: Risk factors for preeclampsia

Diabetes mellitus
Renal disease
History of preeclampsia with previous pregnancies
Extremes of maternal age
Nulliparity
Multiple gestations
Family history of preeclampsia
Chronic hypertension (either essential or secondary)
Thrombophilias
Trophoblastic disease (i.e., hydatidiform mole)
Autoimmune and connective tissue diseases

Diabetes mellitus

Renal disease

History of preeclampsia with previous pregnancies

age

Nulliparity

Multiple gestations

Family history of preeclampsia

Chronic hypertension (either essential or secondary)

Thrombophilias

Autoimmune and connective tissue diseases has led to several theories implicating structural and/ or functional changes in the developing placenta as factors contributing to preeclampsia. The failure of the placental spiral arteries to lose their musculoelastic layers, ultimately leading to decreased placental perfusion, was considered to be the central pathophysiological event in preeclampsia for decades. ${ }^{5,6}$ Placental hypoxia ensues following the decrease in placental perfusion, resulting in placental production of vasoactive soluble factors, which, when released into the maternal circulation, result in endothelial dysfunction and clinical features of preeclampsia. ${ }^{7}$ Over the last decade, preeclampsia has been associated with elevated levels of the soluble receptor for vascular endothelial growth factor (VEGF) of placental origin. ${ }^{8}$ This soluble receptor, commonly referred to as soluble fms-like tyrosine kinase receptor-1, may bind and neutralize VEGF and limit the availability of free VEGF for placental angiogenesis, thus representing the missing link between placental ischemia and maternal endothelial dysfunction. However, this mechanism may not necessarily account to a similar extent for all cases of preeclampsia, as many pathophysiological processes that ultimately result in endothelial dysfunction may contribute to its pathophysiology. ${ }^{9}$ Notably, an imbalance between vasodilatory and vasoconstricting prostaglandins, favoring the latter, may be a contributing factor, ${ }^{10}$ as well as the presence of agonistic antibodies to angiotensin $\mathrm{II}_{1}^{11}$ reduced heme oxygenase- 1 levels, ${ }^{12}$ nitric oxide dysfunction, ${ }^{13}$ and upregulation of several vasoactive mediators, including cellular fibronectin, ${ }^{14}$ von Willebrand factor, ${ }^{15}$ cell adhesion molecules, ${ }^{16,17}$ and cytokines. $^{18,19}$

\section{Preeclampsia is a Heterogeneous Disease}

It has been increasingly recognized that preeclampsia is a heterogeneous disease, with the different clinical subtypes possibly reflecting distinct underlying pathological mechanisms. ${ }^{9,20}$ It is common in clinical practice, e.g., to subcategorize preeclampsia as early versus late onset (before and after 34 weeks of gestation respectively), ${ }^{21}$ with early preeclampsia commonly presenting with severe features, including thrombocytopenia, impaired liver function tests, the new development of renal insufficiency, pulmonary edema, or new-onset cerebral or visual disturbances. $^{2}$

Recent evidence suggests that women with early, severe preeclampsia may have a more pronounced antiangiogenic imbalance and less favorable outcome than those with late preeclampsia. ${ }^{22,23}$ Early preeclampsia can also further be viewed as placental preeclampsia, as it is commonly associated with poor placentation, the pathological substrate for fetal growth restriction. ${ }^{24}$ In contrast, late preeclampsia is not associated with 
abnormal placentation and the associated decreased perfusion. This late form occurs in women with vascular dysfunction that is present prior to pregnancy (commonly due to hypertension, diabetes, or obesity), in whom pregnancy acts as a physiological stress that exacerbates preexisting vascular inflammation and endothelial dysfunction (i.e., maternal preeclampsia).

The dichotomous view of preeclampsia (e.g., early, severe, placental versus late, mild, maternal) is likely overly simplistic, and it is more likely that there is an overlap between maternal preexisting systemic inflammation (such as that which is present in obesity) and impaired angiogenesis. Further understanding of the relative contributions of inflammation, antiangiogenesis, or other mechanisms to the different subtypes of preeclampsia may be the first step toward developing targeted therapies. Of note, several therapeutic/preventive strategies have been studied in preeclampsia. None has been applicable to all patients, likely due to the failure to identify the preeclampsia subtype and to provide targeted treatments. ${ }^{23,25}$

Over the last five decades, much progress has been made in improving blood pressure control in preeclampsia and in the prevention of eclamptic seizures (see Management). Delivery, however, remains the mainstay of therapy for severe forms and anticipated life-threatening complications. As the severe forms of preeclampsia tend to develop early in pregnancy, labor induction, commonly for maternal indications, usually results in a preterm delivery, with low birth weight and related neonatal complications.

Chronic hypertension in pregnant women is diagnosed based on the presence of hypertension before the 20th week of gestation. ${ }^{1}$ The diagnosis may be difficult in young women with little or no medical history. Physiologic changes during pregnancy lead to a fall in blood pressure during the first and second trimesters. In a woman with undiagnosed hypertension prior to pregnancy, this may lead to normal readings early in pregnancy. During the third trimester, the return to prepregnancy hypertensive values may lead to the false diagnosis of new-onset hypertension.

The epidemiology of chronic hypertension in pregnancy has changed significantly over the last couple of decades. This is predominantly due to a trend toward advanced age at first pregnancy, further confounded by the sophisticated techniques of assisted reproduction, such as in vitro fertilization (IVF), that have made pregnancy possible for women with infertility conditions that are associated with cardiovascular disease (CVD) risk factors (such as polycystic ovary syndrome). The care of these patients imposes two major challenges. First, the classical approach that suggests that pregnant women with chronic hypertension are at low risk for cardiovascular complications within the short duration of pregnancy and, therefore, do not require blood pressure treatment may be suboptimal for this particular group. These women, furthermore, may be at even a greater risk for superimposed preeclampsia, due to preexisting comorbidities (such as diabetes, renal disease, and hypertension). In addition, several studies have reported an increased risk of preeclampsia in pregnancies conceived by IVF. ${ }^{26}$

Up to $30 \%$ of pregnancies in women with chronic hypertension may be affected. In women with chronic hypertension, but no proteinuria at baseline, preeclampsia is heralded by the development of proteinuria after 20 weeks of gestation. Some women may have proteinuria at baseline, and this can significantly complicate the diagnosis. In general, the development of severe hypertension ( $\geq 160 \mathrm{~mm} \mathrm{Hg}$ systolic and/or $\geq 110 \mathrm{~mm} \mathrm{Hg}$ diastolic) or any of the signs or symptoms of severe preeclampsia (Table 3) signify the development of superimposed preeclampsia; these women are at a particularly high risk for cerebral hemorrhage and placental abruption.

Gestational hypertension is characterized by newonset hypertension after the 20th week of gestation, in the absence of proteinuria. ${ }^{1}$ Women who develop gestational hypertension after 30 weeks gestation have a $10 \%$ risk of progressing to preeclampsia; this risk increases to $30 \%$ in women who present before 30 weeks gestation. Women who do not develop proteinuria are diagnosed with either transient hypertension (normalization of blood pressure by 12 weeks postpartum) or chronic hypertension (elevated blood pressure and need for blood pressure treatments that persist after pregnancy and delivery).

During the first five postpartum days, blood pressure in normotensive women tends to be higher compared with

Table 3: Diagnostic criteria for severe preeclampsia Any of these findings:

Severe hypertension Blood pressure $\geq 160$ systolic or $\geq 110$ diastolic on two occasions, 4 hours apart

Renal impairment Serum creatinine $>1.1 \mathrm{mg} / \mathrm{dL}$ or its doubling in the absence of other renal disease

Hepatic involvement ${ }^{*}$ Elevated liver function tests (twice upper limit of normal)

Right upper quadrant pain (unresponsive to medications, or not related to other causes, or both)

Thrombocytopenia* Platelet count $<100 \times 10^{9} / \mathrm{L}$

Neurological signs New onset of cerebral or visual and symptoms ${ }^{* *} \quad$ disturbances

Cardiovascular Pulmonary edema

compromise

*Presence of these findings should prompt consideration of HELLP syndrome

**May represent impending eclampsia 
their pregnancy values, likely due to fluid shifts from the interstitial space and the resultant transient rise in the intravascular volume. While hypertension in most of these women resolves by 2 to 4 weeks postpartum, some of these women may remain hypertensive and require antihypertensive treatment. A subset of these patients may progress to preeclampsia/eclampsia that, due to the onset of hypertension after delivery (i.e., postpartum preeclampsia), may represent a diagnostic challenge leading to delayed treatment. An increasing awareness of this clinical entity led to a new set of recommendations by the College advising close blood pressure monitoring in the hospital and outpatient surveillance up to 10 days postpartum of women with gestational hypertension. ${ }^{2}$ In addition, all women should receive discharge instructions regarding signs and symptoms of preeclampsia and the need to see their health care providers immediately should signs/ symptoms develop. Treatment of postpartum preeclampsia relies on blood pressure control and magnesium sulfate administration for prevention of seizures.

At the mechanistic level, postpartum preeclampsia is particularly interesting as it occurs in the absence of the placenta, thus challenging one of the widely accepted concepts in the pathogenesis of preeclampsia: The role of the placenta as the major culprit. Postpartum preeclampsia likely represents a clinical subtype with a distinct underlying mechanism; its clinical presentation further supports the evolving concept of preeclampsia being a heterogeneous disease rather than a single clinical entity.

The HELLP syndrome is believed to be a deceptive form of preeclampsia, which presents with the distinctive and ominous triad of microangiopathic hemolytic anemia, hepatocellular injury, and consumption of platelets. ${ }^{3}$ Right upper quadrant and epigastric pain may occur as a consequence of a hepatic hematoma and resultant stretching of Glisson's capsule. It may herald hepatic rupture, which is associated with high maternal and fetal mortality rates. Urgent delivery remains the mainstay of treatment for patients with HELLP syndrome. Retrospective analyses of HELLP syndrome patients, in whom urgent delivery was not undertaken, have documented an extremely high perinatal infant mortality of $70 \% .^{27}$

Eclampsia refers to the development of seizures in a woman with preeclampsia or gestational hypertension. While the classic presentation is the development of seizures in a woman with severe preeclampsia, up to $20 \%$ of cases occur in women with no evidence of proteinuria, and many cases occur in patients with mild hypertension. The mainstay of treatment is intravenous magnesium sulfate, which has been shown to be more effective than either phenytoin or diazepam for seizure prophylaxis in women with severe preeclampsia and for prevention of recurrent seizures in those with eclampsia. ${ }^{28}$

\section{MANAGEMENT OF HYPERTENSIVE PREGNANCY DISORDERS}

The goal for the treatment of hypertensive pregnancy disorders is to prevent maternal complications without compromising fetal well-being and safety. The factors that affect management decisions are maternal, including the duration and severity of hypertension and the presence of end-organ damage, as well as fetal, such as safety of blood pressure medications and the potential for uteroplacental and fetal circulation compromise due to overzealous blood pressure control. The following discussion will address preventive strategies, preconception counseling, blood pressure treatment goals and options, and timing of delivery.

\section{Preeclampsia Prevention}

Several different strategies have been evaluated for the prevention of preeclampsia, including a low-salt diet, diuretics, low-dose aspirin, antioxidants, and calcium and magnesium supplements. With the exception of calcium supplementation for calcium-deficient women, and low-dose aspirin for women at high risk (i.e., those with a history of chronic hypertension and preeclampsia in a previous pregnancy), ${ }^{29}$ these approaches have failed to provide reproducible benefits.

\section{Preconception Counseling}

Women with a history of hypertension should be evaluated before pregnancy for target organ damage, such as left ventricular hypertrophy, hypertensive nephropathy, and retinopathy, which will help establish blood pressure treatment goals. Patients with clinical clues suggestive of secondary hypertension (e.g., hard-to-control hypertension requiring more than three antihypertensive agents and/or indicative laboratory and clinical findings) should undergo a workup for secondary hypertension (primary hyperaldosteronism, pheochromocytoma, and renal artery stenosis). Therefore, prepregnancy counseling of women with chronic hypertension may require expertise outside the realm of obstetrics. Some forms of secondary hypertension, particularly pheochromocytoma and renovascular hypertension, may further increase the risk for adverse pregnancy outcomes beyond that of essential hypertension; thus, surgery (for pheochromocytoma) or revascularization (for renal artery stenosis) should be considered before pregnancy. In this population of young women of childbearing age, these interventions may result in cure of hypertension. The prepregnancy evaluation should also address changes in medications to those that have acceptable safety profiles in pregnancy and include counseling related to pregnancy risks. It is estimated that as many as $25 \%$ of women with chronic 
hypertension may develop superimposed preeclampsia. These women are at a particularly high risk for cerebral hemorrhage and placental abruption. It remains unclear whether early treatment of chronic hypertension in pregnancy prevents preeclampsia. ${ }^{30}$ In the absence of randomized prospective trials adequately powered to address this important clinical question, practicing physicians should treat chronically hypertensive women according to currently accepted national guidelines, which are presented and discussed below.

\section{Blood Pressure Treatment Goals and Options}

It is useful to divide hypertensive pregnancy disorders into two general categories for purposes of optimizing hypertension evaluation and treatment. The first includes the acute hypertensive syndromes of preeclampsia/ eclampsia/HELLP syndrome, which carry a high-risk for maternal and fetal morbidity and mortality. The most important reason for the initiation of antihypertensive treatment in these patients is to prevent maternal cerebrovascular and cardiac complications. While hypertension in these settings can be treated medically, the definitive treatment remains delivery. The second general category is chronic hypertension. Ideally, these women should be evaluated before pregnancy, which will help establish blood pressure treatment goals (see Preconception Counseling). The treatment of gestational hypertension depends upon whether proteinuria subsequently develops. In the absence of proteinuria, blood pressure either returns to normal by 12 weeks postpartum (transient hypertension) or fails to normalize, leading to the diagnosis of chronic hypertension. Women with gestational hypertension who subsequently develop proteinuria are then considered to have developed preeclampsia and are treated accordingly.

\section{Hypertension in Preeclamptic Patients}

The most important reason to initiate antihypertensive treatment in these patients is to prevent maternal cerebrovascular and cardiac complications. While hypertension in these settings can be treated medically, the definitive treatment remains delivery.

The most recent College guidelines ${ }^{2}$ suggest medical therapy for severe hypertension (sustained systolic blood pressures of at least $160 \mathrm{~mm} \mathrm{Hg}$ and/or sustained diastolic blood pressures of at least $110 \mathrm{~mm} \mathrm{Hg}$ ) in order to decrease the incidence of maternal cardiac and cerebral events. The medications most commonly used for urgent control of hypertension include intravenous hydralazine and labetalol. Medications that may be used, along with their safety profiles and dosing schedules, are summarized in Tables 4 and 5, respectively. Control of blood pressure does not cure preeclampsia or prevent its progression: Eclamptic seizures can occur when the blood pressure is only mildly elevated. Therefore, in addition to antihypertensive therapy, preeclamptic patients should receive seizure prophylaxis with intravenous magnesium sulfate, which should be continued during labor and delivery, and for at least 24 hours after delivery. As magnesium is renally excreted, the rate of continuous

Table 4: Classes and specific medications useful for hypertension in pregnancy

\begin{tabular}{|c|c|c|c|}
\hline & & Benefits & Risks \\
\hline \multicolumn{4}{|l|}{ Central agents } \\
\hline Preferred & Methyldopa & Proven safety and efficacy & Neurodepressant side effects \\
\hline Alternative & Clonidine & Efficacy similar to methyldopa & Unproven safety \\
\hline \multicolumn{4}{|l|}{ Beta blockers } \\
\hline Preferred & Labetalol & $\begin{array}{l}\text { Safety and efficacy similar to } \\
\text { methyldopa. May be used for } \\
\text { hypertensive urgency }\end{array}$ & $\begin{array}{l}\text { Fetal bradycardia, neonatal } \\
\text { hypoglycemia, decreased uteroplacental } \\
\text { flow }\end{array}$ \\
\hline Contraindicated & Atenolol & None compared with labetalol & Intrauterine growth restriction \\
\hline \multicolumn{4}{|c|}{ Calcium channel blockers } \\
\hline Preferred & Nifedipine & $\begin{array}{l}\text { Lowers blood pressure without } \\
\text { affecting umbilical artery flow }\end{array}$ & $\begin{array}{l}\text { Fetal distress, profound hypotension with } \\
\text { magnesium }\end{array}$ \\
\hline Alternative & Verapamil & Similar efficacy to other oral agents & $\begin{array}{l}\text { Untested safety profile, risk of interaction } \\
\text { with magnesium }\end{array}$ \\
\hline \multicolumn{4}{|c|}{ Direct vasodilators } \\
\hline Preferred & Hydralazine & Most efficacious oral agent & $\begin{array}{l}\text { Maternal neuropathy, drug-induced lupus, } \\
\text { neonatal thrombocytopenia, and lupus }\end{array}$ \\
\hline Alternative & Nitroprusside & Effective in severe hypertension & Cyanide and thiocyanate toxicity \\
\hline \multicolumn{4}{|l|}{ Diuretics } \\
\hline Preferred & Thiazide & $\begin{array}{l}\text { Useful in chronic HTN, renal } \\
\text { failure, HF }\end{array}$ & $\begin{array}{l}\text { Volume contraction, electrolyte } \\
\text { abnormalities }\end{array}$ \\
\hline Contraindicated & Spironolactone & None & Possible fetal antiandrogen effects \\
\hline
\end{tabular}

HTN: Hypertension; HF: Heart failure 
Table 5: Antihypertensive therapies of acute and chronic hypertension during pregnancy

\begin{tabular}{|c|c|c|}
\hline Indication & Specific drug & Dose \\
\hline \multirow{2}{*}{$\begin{array}{l}\text { Urgent blood } \\
\text { pressure } \\
\text { control }\end{array}$} & Labetalol & $\begin{array}{l}20 \text { to } 80 \mathrm{mg} \text { IV every } 10 \mathrm{~min} \text {, } \\
\text { maximum of } 220 \mathrm{mg}\end{array}$ \\
\hline & Hydralazine & $\begin{array}{l}5 \text { to } 10 \mathrm{mg} \text { IV or IM every } \\
20 \mathrm{~min} \text {, if no success } \\
\text { by } 30 \mathrm{mg} \text { total, consider } \\
\text { alternatives }\end{array}$ \\
\hline \multirow{5}{*}{$\begin{array}{l}\text { Chronic blood } \\
\text { pressure } \\
\text { control }\end{array}$} & Methyldopa & $\begin{array}{l}\text { Initial } 250 \text { mg oral TID, } \\
\text { max } 3,000 \text { mg daily }\end{array}$ \\
\hline & Labetalol & $\begin{array}{l}100 \mathrm{mg} \text { oral BID, titrate to } \\
600 \mathrm{mg} \text { BID }\end{array}$ \\
\hline & Hydralazine & $\begin{array}{l}\text { Initiate } 10 \mathrm{mg} \text { QID, titrate } \\
\text { to } 50 \mathrm{mg} \text { QID, maximum } \\
\text { of } 300 \mathrm{mg}\end{array}$ \\
\hline & Hydrochlorothiazide & 12.5 to $25 \mathrm{mg}$ orally daily \\
\hline & Nifedipine ER & $\begin{array}{l}\text { Initial } 30 \text { to } 60 \mathrm{mg} \text { oral } \\
\text { daily, max } 120 \mathrm{mg} \text { daily }\end{array}$ \\
\hline
\end{tabular}

IV: Intravenous; IM: Intramuscular; TID: Thrice a day; BID:

Twice a day; QID: Four Times a day

infusion, but not the loading dose, should be adjusted, i.e., decreased in women with renal failure. Serum magnesium levels should also be checked more frequently (every 1-2 hours) in these women, compared with women with normal renal function (every 4-6 hours). For women with severe preeclampsia at $<34$ gestational weeks, corticosteroids should be administered to accelerate fetal lung development. Delivery can be deferred for 48 hours for stable patients and in the absence of fetal compromise.

\section{Chronic Hypertension in Pregnancy}

Medications prescribed before pregnancy in women with chronic hypertension can be continued during pregnancy (Tables 4 and 5), except angiotensin-converting enzyme inhibitors, angiotensin II receptor blockers, renin inhibitors, atenolol, and spironolactone. According to the College recommendations, for pregnant patients with chronic hypertension, antihypertensive therapy is recommended for a systolic pressure $\geq 160 \mathrm{~mm} \mathrm{Hg}$ and/ or diastolic blood pressure $\geq 105 \mathrm{~mm} \mathrm{Hg}$. It is suggested that blood pressure levels be maintained between 120/80 and 160/105 $\mathrm{mm} \mathrm{Hg}$.

Thresholds for blood pressure treatment are higher for pregnant than for nonpregnant patients due to a lack of studies to support the benefit of treatment for mild diastolic hypertension (90-99 $\mathrm{mm} \mathrm{Hg}$ ) and concerns for fetal safety, as treatment-induced blood pressure drops were shown to be associated with impaired fetal growth in a meta-analysis of published studies. ${ }^{31}$ It is important to notice that the threshold which was set for the initiation of antihypertensive therapy does not reflect common clinical practice, as antihypertensive therapy is usually instituted for a systolic pressure $\geq 150 \mathrm{~mm} \mathrm{Hg}$ and/or diastolic blood pressure $\geq 100 \mathrm{~mm} \mathrm{Hg} .{ }^{32}$ Another exception is made in women with evidence of end-organ damage: In the presence of renal disease, proteinuria, left ventricular hypertrophy, and retinopathy, antihypertensive therapy is commonly initiated for a diastolic blood pressure $\geq 90 \mathrm{~mm} \mathrm{Hg}$. Recent evidence argues that this approach may be beneficial for the mother without having detrimental fetal effects. A study that compared less tight control of hypertension (target diastolic blood pressure of $100 \mathrm{~mm} \mathrm{Hg}$ ) vs tight control ( $85 \mathrm{~mm} \mathrm{Hg})$, among pregnant women with either gestational or chronic hypertension, showed that tight control of hypertension in pregnancy conferred no apparent benefit to the fetus, but also did not pose a risk to the fetus or newborn. ${ }^{33}$ Tight control showed moderate benefit in preventing progression to severe hypertension in the mother. This finding is supported further by a meta-analysis that included 49 trials (with 4,723 patients), which showed that antihypertensive therapy for mild-tomoderate hypertension in pregnancy reduced the risk (by half) of developing severe hypertension. ${ }^{30}$

\section{Timing of Delivery}

Hypertensive pregnant patients are typically cared for by high-risk obstetricians, and the decision to proceed with delivery is made only after a careful assessment of risks to the fetus and mother. Input provided by internists and related subspecialties increasingly is being recognized as important for the optimization of patient blood pressure treatment and pregnancy outcomes.

Consideration commonly is given to postponing delivery in pregnancies affected by preeclampsia before 34 weeks of gestation, ${ }^{2}$ as the fetus is still immature and may suffer profound consequences due to incomplete respiratory development. This approach is only reasonable in cases in which the maternal risk is relatively low. Induction of labor should be entertained in circumstances of adequate fetal development or progression to severe forms of hypertensive disease, irrespective of fetal maturity. The indications for urgent delivery include uncontrollable severe hypertension, eclampsia, pulmonary edema, placental abruption, and fetal distress.

\section{HYPERTENSIVE PREGNANCY DISORDERS AND FUTURE CARDIOVASCULAR HEALTH}

The cardiovascular complications of hypertensive pregnancy disorders traditionally were believed to be confined to pregnancy. In recent years, however, epidemiological studies consistently have demonstrated an association between an increased risk for CVD in adulthood and low birth weight, ${ }^{34}$ which, in children born to preeclamptic mothers, may occur as a consequence of either prematurity or intrauterine growth restriction. 
In addition, women with a history of hypertensive compared with normotensive pregnancies are at increased risk for the development of hypertension, ischemic heart disease, or stroke later in life. ${ }^{35}$ The highest risk was reported for women with severe forms of preeclampsia who delivered prematurely. The likely mechanism for this association is that these two conditions share several common risk factors, such as renal disease and diabetes mellitus, which may lead to hypertensive pregnancy disorders and CVD at different times in a woman's life. Thus, a history of hypertensive pregnancy disorders may aid in identifying women at risk for future CVD. Primary prevention in these women should focus on lifestyle modifications (exercise, weight loss, and smoking cessation), early detection of CVD risk factors, and treatment according to evidence-based national guidelines.

\section{CONCLUSION}

Hypertensive pregnancy disorders remain a major therapeutic challenge. Their etiologies and underlying pathophysiologies remain poorly understood, thus not allowing for targeted therapeutic approaches. Emerging evidence argues against several postulates that are commonly used to justify different approaches to treatment of hypertension in pregnancy compared with hypertension in the general population. First, the prevailing view that hypertensive pregnant women are at low risk for cardiovascular complications within the short duration of pregnancy may not apply to women with advanced age at first pregnancy (a trend increasingly described in many countries) and/or those who underwent fertility treatments for the conditions that are associated with increased CVD risks. Second, despite the lack of studies to indicate that treatment for mild hypertension is beneficial for the fetus, recent data suggest that it prevents progression to severe hypertension in the mother, without posing a risk to the newborn. This recent evidence sets the stage for future studies that should be powered adequately to explore the effects of tight blood pressure control in subgroups of women with preexisting CVD risks, including those with advanced age at pregnancy. In addition, as hypertensive pregnancy disorders have been associated with future CVD, it is plausible that better blood pressure control during pregnancy may improve future cardiovascular outcomes. Additional studies in this developing field are needed that may lead to improvements in both pregnancy and long-term outcomes.

\section{REFERENCES}

1. Report of the National High Blood Pressure Education Program working group on high blood pressure in pregnancy. Am J Obstet Gynecol 2000 Jul;183(1):S1-S22.
2. Hypertension in pregnancy. Report of the american college of obstetricians and gynecologists' task force on hypertension in pregnancy. Obstet Gynecol 2013 Nov;122(5):1122-1131.

3. Jones SL. HELLP! A cry for laboratory assistance: a comprehensive review of the HELLP syndrome highlighting the role of the laboratory. Hematopathol Mol Hematol 1998;11 (3-4):147-171.

4. MacKay AP, Berg CJ, Atrash HK. Pregnancy-related mortality from preeclampsia and eclampsia. Obstet Gynecol 2001 Apr;97(4):533-538.

5. Khong TY, De Wolf F, Robertson WB, Brosens I. Inadequate maternal vascular response to placentation in pregnancies complicated by pre-eclampsia and by small-for-gestational age infants. Br J Obstet Gynaecol 1986 Oct;93(10):1049-1059.

6. Meekins JW, Pijnenborg R, Hanssens M, McFadyen IR, van Asshe A. A study of placental bed spiral arteries and trophoblast invasion in normal and severe pre-eclamptic pregnancies. Br J Obstet Gynaecol 1994 Aug;101(8):669-674.

7. Genbacev O, Zhou Y, Ludlow JW, Fisher SJ. Regulation of human placental development by oxygen tension. Science 1997 Sep;277(5332):1669-1672.

8. Maynard SE, Min JY, Merchan J, Lim KH, Li J, Mondal S, Libermann TA, Morgan JP, Sellke FW, Stillman IE, et al. Excess placental soluble fms-like tyrosine kinase 1 (sflt1) may contribute to endothelial dysfunction, hypertension, and proteinuria in preeclampsia. J Clin Invest 2003 Mar;111(5):649-658.

9. Powers RW, Roberts JM, Plymire DA, Pucci D, Datwyler SA, Laird DM, Sogin DC, Jeyabalan A, Hubel CA, Gandley RE. Low placental growth factor across pregnancy identifies a subset of women with preterm preeclampsia: Type 1 versus type 2 preeclampsia? Hypertension 2012 Jul;60(1):239-246.

10. Mills JL, DerSimonian R, Raymond E, Morrow JD, Roberts LJ 2nd, Clemens JD, Hauth JC, Catalano P, Sibai B, Curet LB, et al. Prostacyclin and thromboxane changes predating clinical onset of preeclampsia: a multicenter prospective study. JAMA 1999 Jul;282(4):356-362.

11. Wallukat G, Homuth V, Fischer T, Lindschau C, Horstkamp B, Jüpner A, Baur E, Nissen E, Vetter K, Neichel D, et al. Patients with preeclampsia develop agonistic autoantibodies against the angiotensin at1 receptor. J Clin Invest 1999 Apr;103(7): 945-952.

12. Baum M, Schiff E, Kreiser D, Dennery PA, Stevenson DK, Rosenthal T, Seidman DS. End-tidal carbon monoxide measurements in women with pregnancy-induced hypertension and preeclampsia. Am J Obstet Gynecol 2000 Oct;183(4): 900-903.

13. Lowe DT. Nitric oxide dysfunction in the pathophysiology of preeclampsia. Nitric Oxide 2000 Aug;4(4):441-458.

14. Islami D, Shoukir Y, Dupont P, Campana A, Bischof P. Is cellular fibronectin a biological marker for pre-eclampsia? Eur J Obstet Gynecol Reprod Biol 2001 Jul;97(1):40-45.

15. Friedman SA, Schiff E, Emeis JJ, Dekker GA, Sibai BM. Biochemical corroboration of endothelial involvement in severe preeclampsia. Am J Obstet Gynecol 1995 Jan;172(1 Pt 1):202-203.

16. Halim A, Kanayama N, el Maradny E, Nakashima A, Bhuiyan AB, Khatun S, Terao T. Plasma p selectin (gmp-140) and glycocalicin are elevated in preeclampsia and eclampsia: their significances. Am J Obstet Gynecol 1996 Jan;174(1 Pt 1): 272-277.

17. Krauss T, Kuhn W, Lakoma C, Augustin HG. Circulating endothelial cell adhesion molecules as diagnostic markers for the early identification of pregnant women at risk for 
development of preeclampsia. Am J Obstet Gynecol 1997 Aug;177(2):443-449.

18. Greer IA, Lyall F, Perera T, Boswell F, Macara LM. Increased concentrations of cytokines interleukin- 6 and interleukin-1 receptor antagonist in plasma of women with preeclampsia: a mechanism for endothelial dysfunction? Obstet Gynecol 1994 Dec;84(6):937-940.

19. Vince GS, Starkey PM, Austgulen R, Kwiatkowski D, Redman CW. Interleukin-6, tumour necrosis factor and soluble tumour necrosis factor receptors in women with pre-eclampsia. Br J Obstet Gynaecol 1995 Jan;102(1):20-25.

20. Ness RB, Roberts JM. Heterogeneous causes constituting the single syndrome of preeclampsia: a hypothesis and its implications. Am J Obstet Gynecol 1996 Nov;175(5):1365-1370.

21. Raymond D, Peterson E. A critical review of early-onset and late-onset preeclampsia. Obstet Gynecol Surv 2011 Aug;66(8):497-506.

22. Rana S, Powe CE, Salahuddin S, Verlohren S, Perschel FH, Levine RJ, Lim KH, Wenger JB, Thadhani R, Karumanchi SA. Angiogenic factors and the risk of adverse outcomes in women with suspected preeclampsia. Circulation 2012 Feb;125(7):911-919.

23. Staff AC, Benton SJ, von Dadelszen P, Roberts JM, Taylor RN, Powers RW, Charnock-Jones DS, Redman CW. Redefining preeclampsia using placenta-derived biomarkers. Hypertension 2013 May;61(5):932-942.

24. Xiong X, Demianczuk NN, Saunders LD, Wang FL, Fraser WD. Impact of preeclampsia and gestational hypertension on birth weight by gestational age. Am J Epidemiol 2002 Feb;155(3):203-209.

25. Myatt L, Redman CW, Staff AC, Hansson S, Wilson ML, Laivuori H, Poston L, Roberts JM; Global Pregnancy CoLaboratory. Strategy for standardization of preeclampsia research study design. Hypertension 2014 Jun;63(6):1293-1301.
26. Watanabe N, Fujiwara T, Suzuki T, Jwa SC, Taniguchi K, Yamanobe Y, Kozuka K, Sago H. Is in vitro fertilization associated with preeclampsia? A propensity score matched study. BMC Pregnancy Childbirth 2014 Feb;14(1):1-7.

27. Wagner SJ, Craici IM, Hogan MC, Bailey KR, Garovic VD. The effect of early diagnosis and treatment on maternal and fetal outcomes in patients with hellp syndrome. Biochem Medica 2007 Jun;17(1):60-70.

28. Duley L, Gülmezoglu AM, Henderson-Smart DJ, Chou D. Magnesium sulphate and other anticonvulsants for women with pre-eclampsia. Cochrane Database Syst Rev 2010 Nov;CD000025.

29. Duley L, Henderson-Smart DJ, Meher S, King JF. Antiplatelet agents for preventing pre-eclampsia and its complications. Cochrane Database Syst Rev 2007 Apr;(2):CD004659.

30. Abalos E, Duley L, Steyn DW, DJ. H-S. Antihypertensive drug therapy for mild to moderate hypertension during pregnancy. Cochrane Database Syst Rev 2007 Jan;(1):CD002252.

31. von Dadelszen P, Ornstein MP, Bull SB, Logan AG, Koren G, Magee LA. Fall in mean arterial pressure and fetal growth restriction in pregnancy hypertension: a meta-analysis. Lancet 2000 Jan;355(9198):87-92.

32. August P. Preeclampsia: new thoughts on an ancient problem. J Clin Hypertens (Greenwich) 2000 Mar;2(2):115-123.

33. Magee LA, von Dadelszen P, Rey E, Ross S, Asztalos E, Murphy KE, Menzies J, Sanchez J, Singer J, Gafni A, et al. Less-tight versus tight control of hypertension in pregnancy. N Engl J Med 2015 Jan;372(5):407-417.

34. Barker DJ. Fetal origins of coronary heart disease. BMJ 1995 Jul;311(6998):171-174.

35. Bellamy L, Casas JP, Hingorani AD, Williams DJ. Preeclampsia and risk of cardiovascular disease and cancer in later life: systematic review and meta-analysis. BMJ 2007 Nov;335(7627):974. 